\title{
A view on the mechanism of metalloporphyrin degradation in hydrogen peroxide epoxidation reactions
}

\author{
A.C. Serra ${ }^{\text {a }}$, E.C. Marçalo ${ }^{\text {b }}$, A.M.d'A. Rocha Gonsalves ${ }^{\text {b } *}$ \\ a Universidade Católica Portuguesa, Pólo da Figueira da Foz, Rua Dr. Mendes Pinheiro 24, \\ 3080 Figueira da Foz, Portugal \\ ${ }^{\mathrm{b}}$ Departamento de Química, Faculdade de Ciências e Tecnologia,Universidade de Coimbra, \\ Rua Larga, P-3000 Coimbra, Portugal
}

Received 17 November 2003; accepted 11 January 2004

\begin{abstract}
The stability of biomimetic catalysts is an essential characteristic for them to be of some practical use. The study of the reaction of metalloporphyrin catalysts in hydrogen peroxide oxidations in the presence of benzoic acid allowed to understand whether an intramolecular or intermolecular mechanism is operative in catalyst degradation. Under certain reaction conditions, the presence of a radical inhibitor minimises the catalyst degradation, and therefore, improves the catalytic process.
\end{abstract}

(C) 2004 Elsevier B.V. All rights reserved.

Keywords: Metalloporphyrin; Catalyst; Degradation; Hydrogen peroxide

\section{Introduction}

To increase the applicability of hydrogen peroxide as an oxidant for hydrocarbons still deserves the interest of many researchers [1-3]. More recent contributions to hydrogen peroxide activation by metalloporphyrin catalysts include reactions performed in ionic liquids [4], use of $\beta$ nitrometalloporphyrins [5], and silica supported catalysts [6].

One of the major problems in hydrogen peroxide catalytic oxidations is the metalloporphyrin stability to the reaction conditions. Many studies have simply not considered this problem or circunvented it by performing reactions in the presence of an excess of substrate. However, some recent publications highlight the importance of catalyst stability in hydrogen peroxide oxidations [7-9].

In this study, we look at the problem of catalyst stability in the epoxidation of cis-cyclooctene with hydrogen peroxide, performed in biphasic systems. Two sets of manganese complexes were used as catalysts. Manganese complexes of porphyrins 1, 2 and $\mathbf{3}$ having chlorine (Mn-1), bromine

\footnotetext{
* Corresponding author. Fax: +351-239-826069.

E-mail address: arg@qui.uc.pt (A.M.d. Rocha Gonsalves).
}

(Mn-2) or methyl groups (Mn-3) in the ortho positions of the meso aryl groups are examples of robust catalysts and manganese complexes of porphyrins 4 (Mn-4) and 5 (Mn-5), lacking bulky groups in the ortho position, are low stability catalysts. As a probe for the metalloporphyrin degradation, we performed some of our oxidations in the presence of the zinc complex of porphyrin $\mathbf{5}$ (Zn-5) (Fig. 1).

\section{Results and discussion}

For quite some time, it was more or less generally accepted that a metallo-oxo species (7) was the common intermediate in metalloporphyrin oxidation catalysis. Only after pioneering studies by Watanabe and co-workers $[11,12]$, was the hypothesis of the involvement of the metalloacylperoxo species (6) put forward as a likely active oxidant. Our previous studies presented experimental evidence for the involvement of (6), a species which is favoured in hydrogen peroxide oxidations performed in the presence of an excess of a lipophilic acid as co-catalyst. The manganese-oxo species (7) is the main oxidant species when the oxidation is carried out under low lipophilic acid concentration $[10,13]$. 
<smiles></smiles>

1<smiles>[R]c1c(Cl)cccc1Cl</smiles>
2<smiles>[2H]c1c(Br)cc(Br)c(OC)c1Br</smiles>
3<smiles>[R]c1c(C)cc(C)cc1C</smiles>

4<smiles>[R]c1cccc(OC)c1</smiles>

Fig. 1. Structures of porphyrins.

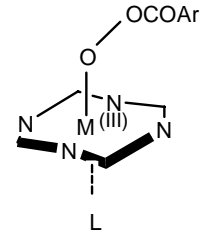

(6)

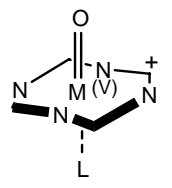

(7)

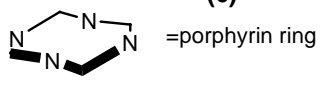

$\mathrm{L}=4$-tert-butylpyridine

In the catalytic process of hydrogen peroxide activation, metalloporphyrins are converted into highly active oxidant species, which, direct or indirectly, can also promote the destruction of the catalyst. Some general ways for the catalyst degradation can be considered [14]. One possibility is the intramolecular decomposition of the highly active species (path a) by a process similar to the described ring destruction in supported metalloporphyrins $[14,15]$. Other processes of macrocycle destruction of intermolecular nature are the oxidation of another molecule of catalyst by the active oxidant species (path b) or the generation of oxygen radical's by interaction with $\mathrm{H}_{2} \mathrm{O}_{2}$, which can destroy the catalyst (path c) $[8,16]$ (Scheme 1).

To determine which type of metalloporphyrin degradation mechanism prevails in catalytic hydrogen peroxide oxidations, distinguishing between the intramolecular or intermolecular mechanism, we added an easily destroyed metalloporphyrin $(\mathbf{Z n}-\mathbf{5})$ to the reaction medium. This experiment allows the identification of an intermolecular mechanism through the control of the stability of $\mathbf{Z n - 5}$. We began our studies by performing the oxidation of cis-cyclooctene in the biphasic system using an excess of a 5\% solution of hydrogen peroxide and catalysts Mn-1 to Mn-5 in the presence of an equimolar amount of $\mathbf{Z n - 5}$. We call condition $\mathrm{A}$, which corresponds to an excess of benzoic acid relatively to the catalyst (20 to 1 ) and condition B that in which an equimolar amount of benzoic acid and catalyst (1:1) was used. Our results are summarised in Table 1.

Table 1 shows that the catalytic process is not affected by the presence of the zinc porphyrin. There is no interaction between the two macrocycles relatively to the catalytic process [17]. We do not detect any significant shift in the spectra of the metalloporphyrins either alone or mixed in solution. Catalytic condition A (excess of benzoic acid) give faster catalysis and originate less catalyst destruction than condition B as pointed out previously by us $[13,18,19]$. Metalloporphyrins without bulky ortho groups in the meso phenyls showed a lower activity as expected [20] but also for these metalloporphyrins, condition A originate higher catalyst stability. Among the metalloporphyrins having bulky ortho groups, those with halogens (Mn-1 and Mn-2) are the best catalysts. The Zn-5 stability depends both on the catalyst structure and reaction conditions. In the presence of catalysts Mn-4 and Mn-5 using either condition A or B, Zn-5 is not destroyed. With Mn-1 the zinc porphyrin $\mathbf{Z n - 5}$ is stable using condition $\mathrm{B}$ but it is degraded using condition A. With Mn-2, and both conditions A and B, Zn-5 is completely destroyed. Using Mn-3 as catalyst, $\mathbf{Z n - 5}$ is more extensively destroyed under condition A than under condition B.

It is clear from the results with catalysts Mn-1 and Mn-3 that two degradation mechanisms are operative. Under condition $\mathrm{B}$, catalyst degradation occurs preferentially by an intramolecular mechanism without destruction of Zn-5 (entries 2 and 8). This same intramolecular degradation mechanism is operative with the poorly active catalyst Mn-4 (entries 9 and 10) and with catalyst Mn-5 under condition B (entry 12). However, under condition A, metalloporphyrins Mn-1 and Mn-3 experiment an intermolecular mechanism of degradation as shown by an efficient destruction of the zinc porphyrin (entries 1 and 7).

In the intermolecular destruction mechanism the attack of the oxidation species must be electrophilic, as became apparent when the zinc complex (Zn-5) was changed for the

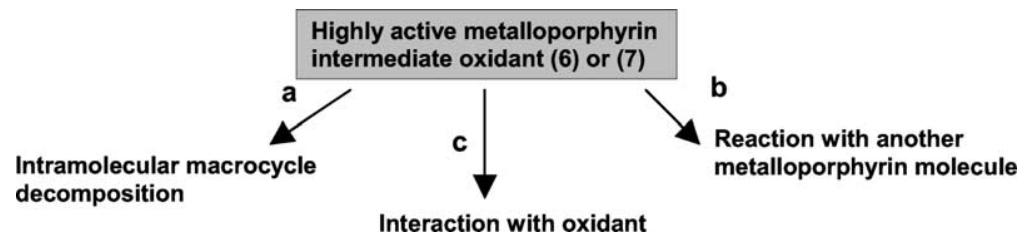

Scheme 1 
Table 1

Results of the cis-cyclooctene epoxidation by catalysts Mn-1 to Mn-5 and the reaction stability of Zn-5

\begin{tabular}{|c|c|c|c|c|c|}
\hline Entry & Catalyst & $\begin{array}{l}\text { Catalytic } \\
\text { conditions }^{\text {a }}\end{array}$ & $\begin{array}{l}\text { Cis-cyclooctene conversion } \\
\text { percentage }(\mathrm{min})\end{array}$ & $\begin{array}{l}\text { Catalyst stability } \\
\text { percentage }(\mathrm{min})^{\mathrm{b}}\end{array}$ & $\begin{array}{l}\text { Zn-5 stability percentage } \\
(\min )^{\mathrm{b}}\end{array}$ \\
\hline 1 & Mn-1 & A & 75 (10), $100(20)$ & $92(20)$ & $31(10), 8$ (20) \\
\hline 2 & Mn-1 & $\mathrm{B}$ & $30(10), 46(20), 58(40)$ & $59(10), 54(20), 50(40)$ & $100(10), 102(20), 104(40)$ \\
\hline 3 & Mn-1 $\mathbf{1}^{\mathrm{c}}$ & A & $92(10), 100(20)$ & $100(20)$ & - \\
\hline 4 & Mn-2 & A & $100(10)$ & $100(10)$ & $0(20)$ \\
\hline 5 & Mn-2 & B & 97 (10), $100(20)$ & $75(20)$ & $0(20)$ \\
\hline 6 & Mn-2 $2^{c}$ & A & $100(10)$ & $100(10)$ & - \\
\hline 7 & $\mathbf{M n}-3^{\mathrm{d}}$ & A & $24(20), 44(40)$ & 84 (20), 76 (40) & $70(20), 46(40)$ \\
\hline 8 & $\mathbf{M n}-\mathbf{3}^{\mathrm{d}}$ & $\mathrm{B}$ & $14(20), 19(40)$ & $22(20), 19(40)$ & $88(20), 88(40)$ \\
\hline 9 & Mn-4 & A & $24(120)$ & $17(120)$ & $108(120)$ \\
\hline 10 & Mn-4 & $\mathrm{B}$ & $0(30)$ & $0(30)$ & $103(30)$ \\
\hline 11 & Mn-5 & A & $4(120)$ & $100(120)$ & 98 (120) \\
\hline 12 & Mn-5 & B & $2(120)$ & $35(120)$ & $98(120)$ \\
\hline
\end{tabular}

${ }^{\text {a }}$ Condition A: catalyst:4-tert-butylpyridine:benzoic acid:cis-cyclooctene: $\mathrm{H}_{2} \mathrm{O}_{2}(5 \%)=1: 1: 20: 200: 1300$; condition B: catalyst:4-tert-butylpyridine: benzoic acid:cis-cyclooctene: $\mathrm{H}_{2} \mathrm{O}_{2}(5 \%)=1: 1: 1: 200: 1300$.

b Measured relatively to the initial value of the Soret band.

c Without zinc porphyrin.

d Catalyst:4-tert-butylpyridine $=1: 5$.

Table 2

Results of the cis-cyclooctene epoxidation by catalysts Mn-1 and Mn-2 in the presence of porphyrin (5)

\begin{tabular}{|c|c|c|c|c|c|}
\hline Entry & Catalyst & $\begin{array}{l}\text { Catalytic } \\
\text { conditions }^{\mathrm{a}}\end{array}$ & $\begin{array}{l}\text { Cis-cyclooctene conversion } \\
\text { percentage }(\mathrm{min})\end{array}$ & $\begin{array}{l}\text { Catalyst stability } \\
\text { percentage }(\min )^{b}\end{array}$ & $\begin{array}{l}\text { Porphyrin }(\mathbf{5}) \text { stability } \\
\text { percentage }(\mathrm{min})^{\mathrm{b}}\end{array}$ \\
\hline 13 & Mn-1 & $\mathrm{A}$ & $84(10), 100(20)$ & $100(10), 98(20)$ & $105(10), 102(20)$ \\
\hline 14 & Mn-2 & A & $98(10), 100(20)$ & $100(10), 100(20)$ & $98(10), 93(20)$ \\
\hline 15 & Mn-2 & $\mathrm{B}$ & $93(20), 98(40)$ & $76(20), 73(40)$ & $88(20), 78(40)$ \\
\hline
\end{tabular}

${ }^{a}$ Condition A: catalyst:4-tert-butylpyridine:benzoic acid:cis-cyclooctene: $\mathrm{H}_{2} \mathrm{O}_{2}(5 \%)=1: 1: 20: 200: 1300$; condition B: catalyst:4-tert-butylpyridine: benzoic acid:cis-cyclooctene: $\mathrm{H}_{2} \mathrm{O}_{2}(5 \%)=1: 1: 1: 200: 1300$.

$\mathrm{b}$ Measured relatively to the initial value of the Soret band.

corresponding free base (5). In the cases where we expected intermolecular attacks little or no degradation of (5) occurred (Table 2).

The process of $\mathbf{Z n - 5}$ degradation is not directly promoted by hydrogen peroxide but involves highly active oxidising manganese complex species. A blank catalytic experiment, in the absence of the Mn-complex, did not lead to Zn-5 degradation.

We suggested before that different metalloporphyrin species, metallo-oxo (8) and metalloacylperoxo (9), could be intermediates able to epoxidise alkenes $[10,13,19]$. Recently, studies from different authors strongly suggest that the same kind of intermediates occur in iron porphyrin systems [21-24]. Under condition A the principal oxidative intermediate is (9) (pathway A) while under condition B (8) is the acting oxidative species (pathway B) (Scheme 2).

Under condition B, Mn-1 (see entry 2, Table 1) preferentially follows pathway B with the formation of a metallo-oxo intermediate (8) which has to be, in this case, the oxidising species. This intermediate has enough activity for alkene epoxidation (II), but does not attack the Zn macrocycle (III). At the same time, some catalyst destruction occurs (I) (Scheme 3).

The catalyst degradation has faster kinetics than the bimolecular reaction with $\mathbf{Z n - 5}$. Two pathways are possible for this degradation: intramolecular decomposition [25] or attack of water or hydrogen peroxide molecules to the metallo-oxo intermediate $(\mathbf{8})[7,26]$. The more or less pronounced radical character of $(\mathbf{8})$ can be an important way of distinguishing which pathway is operative.

The process of intramolecular catalyst destruction can involve the metallo-oxo intermediate (8) [20,27,28], but also a metallo-oxo intermediate (10) $[29,30]$ which is formed

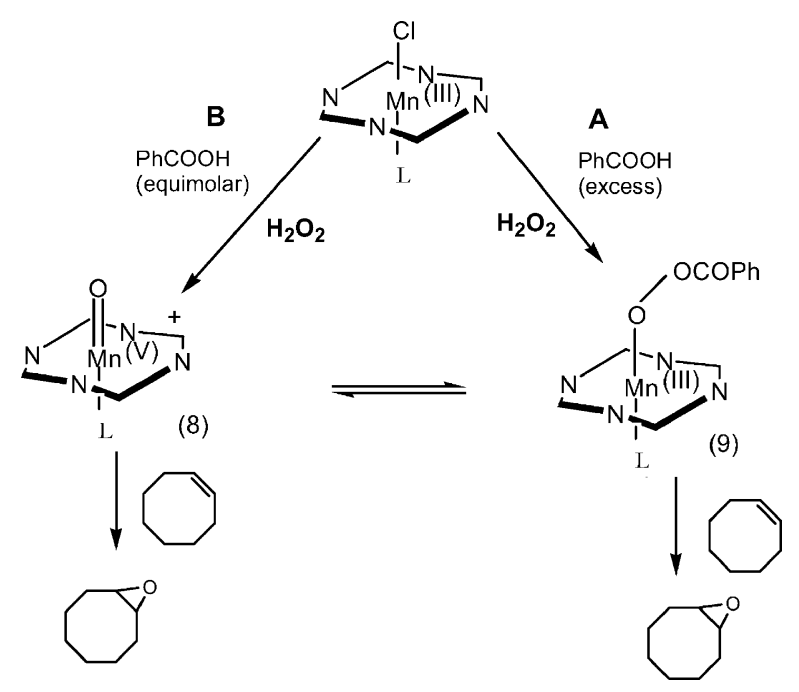

Scheme 2 
Table 3

Catalytic epoxidation of cis-cyclooctene by Mn-2 and Zn-5 stability in the presence of 2,6-di-tert-butyl-4-methoxyphenol as a radical inhibitor

\begin{tabular}{llllll}
\hline Entry & $\begin{array}{l}\text { Inhibitor } \\
\text { ratio }^{c}\end{array}$ & $\begin{array}{l}\text { Catalytic } \\
\text { conditions }^{\text {a }}\end{array}$ & $\begin{array}{l}\text { Cis-cyclooctene conversion } \\
\text { percentage }(\mathrm{min})\end{array}$ & $\begin{array}{l}\text { Catalyst stability } \\
\text { percentage }(\mathrm{min})\end{array}$ & $\begin{array}{l}\text { Zn-5 stability } \\
\text { percentage }(\mathrm{min})\end{array}$ \\
\hline 16 & $1: 20$ & $\mathrm{~B}$ & $93(10), 100(20)$ & $78(10), 75(20)$ & $4(10), 0(20)$ \\
17 & $1: 100$ & B & $56(10), 75(20), 88(40)$ & $100(10), 100(20), 100(40)$ & $108(10), 108(20), 106(40)$ \\
18 & $1: 100$ & A & $74(10), 88(20), 95(40)$ & $100(20), 100(40)$ & $79(20), 21(40)$ \\
\hline
\end{tabular}

${ }^{a}$ Condition A: catalyst:4-tert-butylpyridine:benzoic acid:cis-cyclooctene: $\mathrm{H}_{2} \mathrm{O}_{2}(5 \%)=1: 1: 20: 200: 1300$; condition B: catalyst:4-tert-butylpyridine: benzoic acid:cis-cyclooctene: $\mathrm{H}_{2} \mathrm{O}_{2}(5 \%)=1: 1: 1: 200: 1300$.

${ }^{\mathrm{b}}$ Measured relatively to the initial value of the Soret band.

${ }^{c}$ Molar ratio relatively to catalyst.

from (8) by reaction with hydrogen peroxide [16,31]. Analogous processes have been suggested in iron metalloporphyrin catalysis $[7,8]$ (Scheme 4).

It is possible that the two mechanisms of catalyst destruction occur simultaneously and we cannot predict which is more relevant, although we have evidence that the pathway involving (10) is likely be important. If the reaction corresponding to entry 2 (Table 1 ) is performed in the presence of a radical inhibitor such as 2,6-di-tert-butyl-4-methoxyphenol, in a ratio of 100 to 1 relatively to Mn-1, less catalyst degradation (71\% for Mn-1 final stability) is observed, while cis-cyclooctene conversion is the same $(51 \%)$. It is likely that the radical inhibitor reacts with $(\mathbf{1 0})$ preferentially to $(\mathbf{8})$ regenerating the catalyst and avoiding the intramolecular degradation route [8]. Considering (10) a less active oxidant than (8) [29] the presence of the radical inhibitor does not interfere with the catalytic process. Under condition A Mn-1 proved to be a more efficient catalyst (entry 1 versus entry 2). Pathway $\mathrm{A}$ is the favoured one, a metallo-acylperoxo being the most important epoxidation species. The high reactivity of
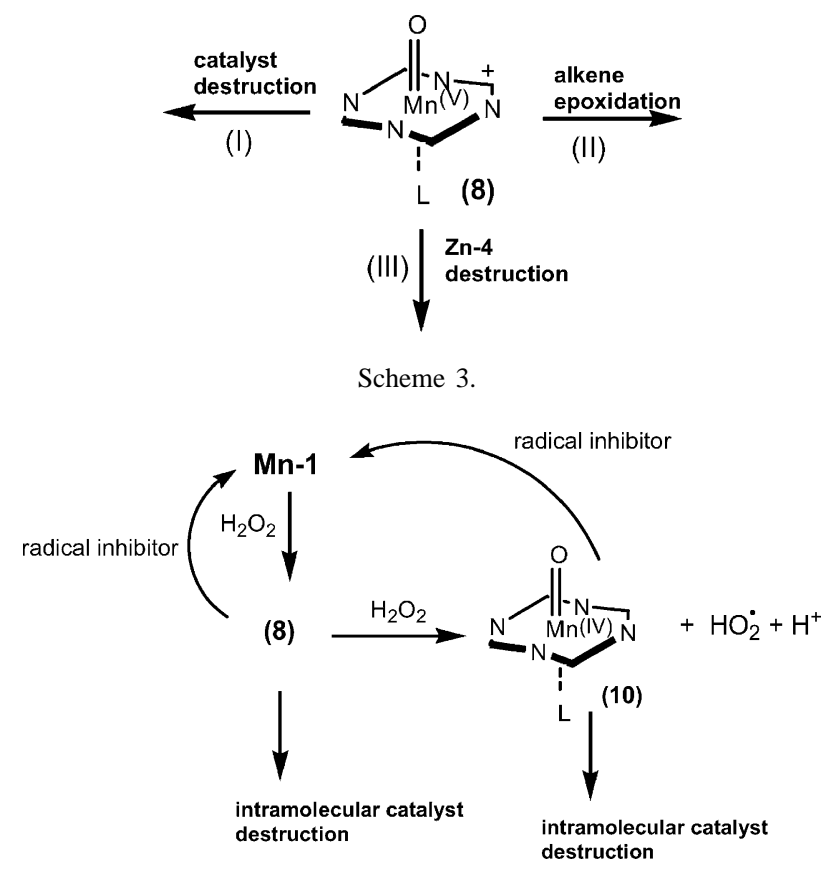

Scheme 4. the metalloacylperoxo species (9) is illustrated by its high rate of catalysis. In this case, pathway $\mathrm{B}$ that originates intramolecular degradation by (8) or (10) is not important, so less catalyst destruction is observed. We suggest that the metallo-acylperoxo generated has enough activity to attack the $\mathbf{Z n - 5}$ structure leading preferentially to an intermolecular degradation of the catalyst.

The catalytic efficiency of Mn-4 and Mn-5 is very low as a consequence of the low activity of the intermediate species responsible for oxidations. In this case, $\mathbf{Z n - 5}$ is not destroyed showing that in the catalytic degradation an intramolecular process must be operative.

For Mn-2 we observed that $\mathbf{Z n - 5}$ is destroyed in both conditions A and B suggesting that the intermolecular degradation mechanism is operative. As shown previously Mn-2 is a more efficient catalyst than Mn-1 [13]. The different behaviour observed relatively to $\mathbf{M n}-\mathbf{1}$, when using condition $\mathrm{B}$, can be explained by the higher activity of the species generated in the catalytic cycle. For Mn-2 under condition B we suggest that the metallo-oxo intermediate $(\mathbf{8})$ has enough activity to attack $\mathbf{Z n - 5}$ as shown in (III) Scheme 3. In order to evaluate this we tried the catalysis in the presence of a radical inhibitor, obtaining results summarised in Table 3.

From these last results we conclude that catalyst degradation and $\mathbf{Z n - 5}$ destruction are stopped in the presence of an excess of the radical inhibitor under condition B. This points again to the reaction of the inhibitor with metalo-oxo species such as (8) or (10) regenerating the initial catalyst, thus preventing the attack of these species on Zn-5 and catalyst destruction(see Scheme 4). Under condition A the destruction of $\mathbf{Z n - 5}$ is not avoided. The main catalytic pathway goes through the metallo-acylperoxo (9) which does not react with the inhibitor, allowing $\mathbf{Z n - 5}$ destruction by intermolecular attack.

\section{Experimental}

${ }^{1} \mathrm{H}$ NMR spectra were recorded on a $300 \mathrm{MHz}$ BrukerAMX spectrometer. Mass spectra were obtained on a VG $7070 \mathrm{E}$ mass spectrometer. Elemental analysis was carried out using a Fisons Instrumental EA 1108-CHNS-0 apparatus. Absorption spectra were measured on a JASCO 7800 spectrophotometer. Gas chromatography was carried out on 
a Hewlett-Packard 5890 A with a flame ionisation detector and equipped with a OV1 $(25 \mathrm{~m} \times 0.3 \mathrm{~mm}$, i.d. $)$ capillary column. GC-MS analyses were made on a Agilent 6890 GC system with a Hewlett-Packard 5973 mass selective detector equipped with a capillary column HP-5 MS (25 m).

Dichloromethane was distilled from $\mathrm{P}_{2} \mathrm{O}_{5}$ before use. Other solvents used were commercially available and used as received. Cis-cyclooctene was obtained from Aldrich and was passed through a short column of alumina before used. Hydrogen peroxide 5\% was prepared from a concentrated solution from Riedel titrated by iodometry. The $\mathrm{pH}$ of this solution was set to 4.5-5 with hydrogen carbonate.

Porphyrins (1), (4) and (5) were prepared by nitrobenzene method [32] and porphyrins $\mathbf{2}$ and $\mathbf{3}$ as described in refs. $[13,33]$. Metalloporphyrin Mn-2 was prepared using manganese acetate in acetic acid [13]. Metalloporphyrins Mn-1, Mn-3, Mn-4 and Mn-5 were prepared by metallation of the corresponding porphyrins by the DMF method [34]. Zn-5 was obtained by methanol/chloroform metallation method.

Catalytic reactions were carried out at room temperature. They were monitored by removing aliquots and examining the products by chromatography. The epoxide of ciscyclooctene was identified by comparison of retention times of an authentic sample and GC-MS experiment. Conversions are reported relatively to bromobenzene as internal standard. All the results correspond to the average of two assays.

Oxidations were carried out as followed: a $20 \mathrm{ml}$ flask is charged with $2.5 \times 10^{-3} \mathrm{mmol}$ of the metalloporphyrin and volumes of dichloromethane solutions of organic acid and axial ligand that gave $2 \mathrm{ml}$ of total volume and the correct organic acid/axial ligand/catalyst ratio. The internal standard was added followed by the alkene and $2 \mathrm{ml}$ of hydrogen peroxide solution $(5 \%)$. The mixture is stirred at maximum rate.

\section{Conclusions}

It was demonstrated that in hydrogen peroxide oxidations, catalyst destruction can occur either by an intramolecular or by an intermolecular mechanism. The structure of the catalyst and mainly the reaction conditions are crucial to establish which of the mechanisms is operative. The alternative degradation mechanisms are related to the structure of the different active intermediates which can be formed in the catalytic cycle, a metallo-oxo species leading to an intramolecular mechanism and a metalloacylperoxo species favouring one of intermolecular nature.

\section{Acknowledgements}

The authors would like to thank Chymiotechnon, UCP and FCT- POCTI/QUI/43214 for financial support.

\section{References}

[1] K. Meimann, R. Neumann, J. Chem. Soc. Chem. Commun. (2001) 487.

[2] H. Yao, D.E. Richardson, J. Am. Chem. Soc. 122 (2000) 3220.

[3] B.S. Lane, M. Vogt, V.J. DeRose, K. Burgess, J. Am. Chem. Soc. 124 (2002) 11946.

[4] K.A. Srinivas, A. Kumar, S.M.S. Chauhan, J. Chem. Soc. Chem. Commun. (2002) 2456.

[5] J.-F. Bartoli, K. Le Barche-Ozette, M. Palacio, P. Battioni, D. Mansuy, J. Chem. Soc. Chem. Commun. (2001) 1718.

[6] F.S. Vinhado, P.R. Martins, A.P. Masson, D.G. Abreu, E.A. Vidoto, O.R. Nascimento, Y. Iamamoto, J. Mol. Catal. A: Chem. 188 (2002) 141.

[7] I.D. Cunningham, T.N. Danks, K.T.A. O'Connel, P.W. Scott, J. Chem. Soc., Perkin Trans. 2 (1999) 2133.

[8] I.D. Cunningham, T.N. Danks, J.N. Hay, I. Hamerton, S. Gunathilagan, Tetrahedron 57 (2001) 6847.

[9] I.D. Cunningham, T.N. Danks, J.N. Hay, I. Hamerton, S. Gunathilagan, C. Lanczak, J. Mol. Catal. A: Chem. 185 (2002) 25.

[10] A.M.d'A. Rocha Gonsalves, A.C. Serra, J. Mol. Catal. A: Chem. 168 (2001) 25.

[11] Y. Watanabe, Y.K. Yamagushi, I. Morishima, K. Takehira, M. Shimizu, T. Hayakawa, H. Orita, Inorg. Chem. 30 (1991) 2581.

[12] K. Machii, Y. Watanabe, I. Morishima, J. Am. Chem. Soc. 117 (1995) 6691.

[13] A.M.d'A. Rocha Gonsalves, A.C. Serra, J. Chem. Soc., Perkin Trans. 2 (2002) 715

[14] D.R. Leanord, J.R. Lindsay Smith, J. Chem. Soc., Perkin Trans. 2 (1991) 25.

[15] C. Gilmartin, J.R. Lindsay Smith, J. Chem. Soc., Perkin Trans. 2 (1995) 243.

[16] T.G. Traylor, S. Tsuchiya, Y.-S. Byun, C. Kim, J. Am. Chem. Soc. 115 (1993) 2775.

[17] M.L. Merlau, W.J. Grande, J.T. Nguyen, S.T. Hupp, J. Mol. Catal. A: Chem. 156 (2000) 78.

[18] A.M.d'A. Rocha Gonsalves, M.M. Pereira, A.C. Serra, Ann. Quim. Int. Ed. 92 (1996) 375

[19] A.M.d'A. Rocha Gonsalves, A.C. Serra, J. Porphyrins Phthalocyanines 4 (2000) 598

[20] S. Quici, S. Banfi, G. Pozzi, Gazz. Chim. Ital. 123 (1993) 597.

[21] W. Nam, M.H. Lim, H.J. Lee, C. Kim, J. Am. Chem. Soc. 122 (2000) 6641.

[22] W. Nam, S.W. Jin, M.H. Lim, J.Y. Ryu, C. Kim, Inorg. Chem. 41 (2002) 3647.

[23] J.P. Cooman, A.S. Chien, T.A. Eberspacher, J.I. Brauman, J. Am. Chem. Soc. 122 (2000) 11098.

[24] N. Suzuki, T. Higuchi, T. Nagano, J. Am. Chem. Soc. 124 (2002) 9622.

[25] M.J. Nappa, C.A. Tolman, Inorg. Chem. 24 (1985) 4711.

[26] K.R. Bretscher, P. Jones, J. Chem. Soc., Dalton Trans. (1988) 2273.

[27] S. Banfi, F. Montanari, S. Quici, J. Org. Chem. 54 (1989) 1850.

[28] S. Banfi, F. Montanari, S. Quici, Recyl. Trav. Chim. Pays-Bas 109 (1990) 117.

[29] J.T. Groves, M.K. Stern, J. Am. Chem. Soc. 110 (1988) 8628.

[30] R.D. Arasasingham, G.X. He, T.C. Bruice, J. Am. Chem. Soc. 115 (1993) 7985.

[31] T.G. Traylor, F. Xu, J. Am. Chem. Soc. 109 (1987) 6201.

[32] R.A.W. Johnstone, M.L.P.G. Nunes, M.M. Pereira, A.M.d'A. Rocha Gonsalves, A.C. Serra, Heterocycles 43 (1996) 1423.

[33] P. Hoffmann, A. Roberts, B. Meunier, Bull. Soc. Chim. Fr. 129 (1992) 85.

[34] A.D. Adler, F.R. Longo, F. Kampas, J. Kim, J. Inorg. Nucl. Chem. 32 (1970) 2443. 